\title{
A Routing Mechanism Based on the Sensing Relevancies of Source Nodes for Time-Critical Applications in Visual Sensor Networks
}

\author{
Daniel G. Costa ${ }^{1,2,3}$, Luiz Affonso Guedes ${ }^{1}$, Francisco Vasques ${ }^{3}$, Paulo Portugal ${ }^{3}$ \\ ${ }^{1}$ Department of Computing and Automation, Federal University of Rio Grande do Norte, Brazil \\ ${ }^{2}$ Department of Technology, State University of Feira de Santana, Brazil \\ ${ }^{3}$ IDMEC/ISR, Faculty of Engineering, University of Porto, Portugal \\ Email: \{danielgcosta@uefs.br, affonso@dca.ufrn.br, vasques@fe.up.pt,pportugal@fe.up.pt\}
}

\begin{abstract}
Wireless sensor networks may be deployed to retrieve visual information from the monitored field, enriching monitoring and control applications. Whenever a set of cameraenabled sensor nodes are deployed for time-critical monitoring, visual information as still images and video streams may need to reach the sink as soon as possible, requiring a differentiated treating of the network when compared with non-critical visual data. In such way, considering that source nodes may have different sensing relevancies for the application, according to the desired monitoring tasks and the current sensors' poses and fields of view, we propose a delay-aware multihop routing mechanism where higher relevant visual data packets are routed through paths with lower end-to-end delay. As sensor nodes are expected to be energy-constrained, transmitting only highrelevant packets through shorter/faster paths may prolong their lifetime and assure longer time-critical delivering, with low impact to the overall monitoring quality.
\end{abstract}

Keywords - Multihop routing; Energy-efficiency; Sensing relevance; Time-critical transmission; Visual sensor networks.

\section{INTRODUCTION}

Visual sensor networks (VSNs) are composed of resourceconstrained self-organizing nodes where some or all of them are endowed with a low-power camera for a series of innovative multimedia sensing functions [1][2]. Visual information retrieved from the monitored field in the form of video streaming, conventional snapshots, infrared or thermal images can significantly enhance a large set of monitoring applications, besides fostering the development of new ones [3][4]. We can cite surveillance, tracking, disaster monitoring, home automation, industrial control, traffic management and battlefield surveillance as some of the applications addressed by visual sensor networks.

Sensor nodes are expected to be battery-operated, turning energy preservation a major issue. Energy is required for processing and communication procedures, but the communication costs are usually much higher than the computational costs [5][6]. In general, we can expect that the energy consumption is directly related with the amount of information to be transmitted, although other issues as idle listening in wireless communication hardware also play an important role in energy consumption. Thus, a reasonable way to preserve energy is to decrease the packets flow over the network.

The quality of visual sensor networks will be a function of how well an area of interest is viewed by the source nodes [2][7]. And such quality depends on the actual application requirements, which dictate what, when and with which constraints a set of static or moving targets or even an area of interest must be monitored by the deployed source sensors. Therefore, source nodes may have different relevancies for the application, according to the monitoring requirements and the current cameras' poses and fields of view. As visual source nodes follow a directional sensing model where redundancy is uncommon, source nodes can be directly mapped to a relevance level according to the importance of the retrieved visual information for the application [7].

In time-critical monitoring applications, visual information transmitted from high-relevant source nodes will need to reach the sink with minimal end-to-end delay. As more than one transmission path may be available from source nodes to the sink and a single node may route packets from different sources, we propose a delay-aware routing mechanism where the sensing relevance is considered when deciding the transmission path that visual packets must be forwarded to. Doing so, we achieve two distinct objectives: 1) the most relevant information for the application is transmitted with lower end-to-end delay and 2) energy is preserved over paths with lower delay since they will receive less average traffic.

In the proposed approach, low-relevant packets will still be transmitted, but they will flow over the remaining "worse" paths. As such visual data packets are expected to have low impact to performed monitoring [7], the overall application quality is not warmed. Employing the proposed routing approach, the time-critical delivering capability of the network is expected to last for longer, keeping high accordance with the monitoring requirements of the application. We performed simulations to estimate the average end-to-end transmission delay and the energy 
consumption when the proposed mechanism is adopted.

The remainder of this paper is organized as follows. Section II presents the related works. The problem formulation is presented in Section III. Section IV brings the proposed routing approach. Some simulation results are presented in Section V, followed by conclusions and references.

\section{RELATED WORKS}

Different routing approaches have been investigated in recent years for wireless sensor networks, addressing issues as energy-efficiency, delay constraints and data packet prioritization [3][8][9]. We are mainly concerned in surveying optimizations in the way packets are routed in wireless multimedia sensor networks, where the transmission requirements are more stringent when compared with traditional scalar sensor networks.

The work in [10] proposes a routing mechanism where higher relevant packets are routed through more reliable paths, where the path reliability is a function of the expected error rate and node failure. Moreover, authors in [10] consider multiples paths toward the sink to increase the probability that the transmitted visual data packets are received with quality as good as possible. In a different way, it is proposed in [11] the Multi-Level Rate-Oriented Routing (MLRR) mechanism, a routing scheme where lower transmission rates should be associated with the nodes having less residual power energy, potentially prolonging their lifetimes. A context-aware multipath routing scheme is proposed in [12], where the relevance of the encoded data is exploited for path selection. Authors proposed the MPMPS (Multi-Priority Multi-Path Selection) algorithm to find the paths with lower end-to-end delay for multimedia streaming, considering a set of available node-disjoint paths. The original flow is split into image and audio substreams, giving to each resulting substream a particular priority according to the current monitoring being performed. Then, the paths with lower delays are assigned to data packets carrying higher relevant data. Still concerning the media contents, the work in [13] proposes a transmission scheduling scheme for multi-radio and multi-hop wireless networks, where the video contents are considered when selecting the multiple available transmission channels.

The sensing relevancies of source nodes and optimizations based on them are a novel concept that can considerably enhance the performance of visual sensor networks, still keeping high monitoring quality. However, practical exploitation of such concept is not straightforward. We presented an extensive discussion of how to compute and assign sensing relevancies levels to source nodes in [7], outlining some practical exploitations in VSNs.

We propose an innovative routing mechanism where highrelevant packets are routed through paths with lower end-toend delay, but in a different way of [12][13], the sensing relevancies of source nodes are exploited to define the packets' priorities, whatever is the characteristics of the transmitted media. Doing so, energy is preserved over the paths with lower delay, potentially benefiting time-critical visual monitoring applications. As each source node is assigned to a sensing relevance level, we expect that the proposed solution performs better in visual sensor networks than existing approaches, keeping high accordance with the application monitoring requirements.

\section{PROBLEM FORMULATION}

Some monitoring applications may require transmission of crucial visual information with minimal delay. However, packet transmission over the network consumes most part of energy, directly defining the network lifetime. In such way, transmission paths with lower end-to-end delay should only be used for transmission of high-relevant packets, potentially prolonging the time-critical delivering capability of the network. In this section we formulate the transmission delays and energy consumption issues in visual sensor networks, in order to facilitate the analyses of the proposed approach.

\section{A. End-to-end delay}

The transmission delay may be originated from different aspects of wireless communications. Among these aspects, we can highlight the radio operation, the medium access mechanism, the congestion control, the error recovery and the transmission rate. The most usual radio hardware is equipped with a single wireless antenna, and thus the radio must be turned on to receive packets from neighbor nodes. In fact, it may be that the radio is turned on but no packet is being received, what is referred as idle listening. As idle listening may rapidly deplete the energy resources of the nodes, dutycycle protocols are employed to insert sleeping periods into the radio operation [14], but node sleeping may increase the communication delay. Besides this, medium access procedures may result in collision and additional delay, as well as when data corruption requires packet retransmission. At last, higher layer mechanisms for congestion control and error recovery also affect the perceived delay of the communication. Such complex scenario is hard to formulate in a mathematical way, pushing us to adopt some level of simplification or to employ realistic discrete event simulators, as presented in Section V.

For a network composed of $n$ routing paths $P=\left\{p_{1}, \ldots, p_{n}\right\}$, we can expect that each path $p_{i}$ has a end-to-end transmission delay $d_{i}$. Each path is composed of one or more intermediate nodes, and all aforementioned aspects of wireless communications can be presented in a 1-hop wireless link. So, we can roughly state that the end-to-end delay increases when packets have to cross more hops, and if $p_{i}>p_{j}$ in number of hops, we can expect that $d_{i}>d_{j}$. Therefore, we will compare the end-to-end delay of different paths through the number of intermediate nodes that compose the paths, in the same way of [12]. Moreover, as the transmission rate may also impact the communication delay, directing affecting the MAC layer collisions and retransmissions, we can also draw a similar conclusion: for $t_{i}$ as the transmission rate in path $p_{i}$ 
and $t_{j}$ as the transmission rate in path $p_{j}$, if $t_{i}>t_{j}$ and $p_{i}=p_{j}$ in number of hops, we can also expect that $d_{i}>d_{j}$.

\section{B. Energy consumption}

The actual energy consumption in each node due to communication depends on many factors, as the employed radio hardware, the transmission power and the physical and MAC protocols. As a result, mathematical formulations considering specific details of physical and MAC operations are very complex, pushing energy consumption models to incorporate some level of simplification [15][16].

We expect that the transmission rate in a routing path directly impact the end-to-end delay. But does it also impact the energy consumption over the path?

Still images or videos produced by source nodes are expected to be transmitted in small data packets (reducing the error probability [17]) and with the same size, and typically many packets will be necessary to transmit even small pieces of visual data. In order to achieve minimal transmission overhead, full-size packets should be transmitted. We consider that every data packet has the same size in bits, $k$, corresponding to the entire packet (data payload plus physical, MAC, network and application layers protocol headers). The size in bits of all protocol headers in each packet is defined as $z$, resulting in an effective payload area of $(k-z)$ in each data packet. For a network composed of $S$ source nodes, we assume that each source node $s, s=1, \ldots, S$, will transmit $D_{s}$ data packets in $f_{s}$ seconds. Considering either lossless or fully-reliable wireless links, each intermediate node in the path from the source $s$ to the sink will have to relay at least $\left(T / f_{s}\right) \cdot D_{s}$ data packets, for a period of $T$ seconds.

Each path $p_{i}$ is composed of $H_{i}$ intermediate hops, $h=\{0$, ..., $\left.H_{i}\right\}$. The consumed energy to send and receive bits in each intermediate node depends on its transmission power, $P w t_{\left(p_{i} h\right)}$, and the power for bits reception, $P w r_{\left(p_{i} h\right)}$. The time for transmission of 1 bit is $t x_{\left(p_{\dot{v}} h\right)}$, according to the transmission rate. The energy consumption due to transmission from source $s$ in a hop $h$ of path $p_{i}, E t_{\left(p_{i}, h, s\right)}$, may be estimated in a simple way considering the amount of information to be transmitted, as expressed in (1). The same is true for the energy consumed due to packet reception, $\operatorname{Er}_{\left(p_{i}, h, s\right)}$, and the sum of both energy values in all hops results in the energy consumption over the entire path, $E_{\left(p_{i}, s\right)}$.

$$
\begin{aligned}
& E t_{\left(p_{i}, h, s\right)}=\left(\frac{T}{f_{s}}\right) \cdot D_{s} \cdot k \cdot P w t_{\left(p_{i}, h\right)} \cdot t x_{\left(p_{i}, h\right)} \\
& \operatorname{Er}_{\left(p_{i}, h, s\right)}=\left(\frac{T}{f_{s}}\right) \cdot D_{s} \cdot k \cdot P w r_{\left(p_{i}, h\right)} \cdot t x_{\left(p_{i}, h\right)} \\
& E_{\left(p_{i}, s\right)}=\sum_{h=0}^{H_{(p)}+1}\left(E t_{\left(p_{i}, h, s\right)}+E r_{\left(p_{i}, h, s\right)}\right)
\end{aligned}
$$

We could extend the basic formulation in (1) adding the energy costs for transmissions and receptions of ACK messages and for the radio hardware switching among the transmitting, receiving and sleeping modes. Moreover, the average packet error rates and retransmissions could be accounted, as expressed in [18]. However, we are mainly concerned herein with estimation of the energy consumption during transmission over the path, and the amount of information to be transmitted seems to play an important role even with the addition of new variables. In general words, more energy is expected to be consumed when packets have to be transmitted through more intermediate nodes. In such way, energy saving should be achieved when less packets have to transmitted.

Based on these basic formulations, we achieved three basic assumptions: a) shorter paths provide lower end-to-end delay, b) lower transmission rate reduces the transmission delay and c) energy saving is achieved when fewer packets have to cross the routing path. These assumptions support the proposed routing approach and will be validated through realistic simulations.

\section{DELAY-AWARE MULTIPATH ROUTING}

Multihop visual sensor networks are likely to be composed of several source nodes, which may be connected to the sink through more than one transmission path. A single intermediate node may belong to more than one transmission path, depending on the nodes positions after deployment and the available paths discovered by the routing protocol. Those router nodes may be used to route packets employing some policy depending on the adopted optimization approach.

In many cases, source nodes may have different monitoring relevancies for the application, defining a novel global QoS parameter for optimization purposes. In [7], we defined that the sensing relevance of each source node is represented by a numeric value referred as the Sensing Relevance (SR) index, ranging from 0 to 15 . As expressed before, the significance of the retrieved visual data and the corresponding SR depend on the application monitoring tasks and the network configuration after deployment. In [7] the sink is expected to compute the values of SR using some automatic or manual approach, associating each source node to a group of relevance, as presented in Table I. The final SR is locally computed by each source node considering such classification and some hardware characteristics as camera's resolution and processing characteristics.

TABLE I. VALUES FOR SR

\begin{tabular}{|c|l|}
\hline $\mathbf{S R}_{\mathbf{s}}$ & \multicolumn{1}{|c|}{ Associated Group of Relevance } \\
\hline 0 & $\begin{array}{l}\text { Irrelevant. No visual information should be } \\
\text { transmitted. }\end{array}$ \\
\hline $1-4$ & $\begin{array}{l}\text { Low relevance. Applications may need only low- } \\
\text { quality versions of the retrieved visual data. }\end{array}$ \\
\hline $5-10$ & $\begin{array}{l}\text { Medium relevance. Applications can tolerate some } \\
\text { monitoring quality loss. }\end{array}$ \\
\hline $11-14$ & $\begin{array}{l}\text { High relevance. Assigned to source nodes that retrieve } \\
\text { crucial visual information of the monitored field. }\end{array}$ \\
\hline 15 & $\begin{array}{l}\text { Maximum relevance. The retrieved visual information } \\
\text { is highly critical. }\end{array}$ \\
\hline
\end{tabular}


Source nodes transmitting packets over the network have to include their own 4-bit SR value in each transmitted data packet. Although such requirement may incur in additional energy consumption, the actual impact depends on the adopted MAC technology and wireless radio [19], and the inclusion of optimization information in data packets is a common approach largely adopted by the academic community [9].

We assume that a SR is already established to each source node employing any assignment approach defined in [7]. Additionally, we assume that the transmission paths are already created using some routing protocol [8] and that the available paths are known by router nodes. Some routing protocols as [20] can provide information of the number of hops of the path, and this information will be considered during routing since the end-to-end delay will be estimated based only on the number of hops of the paths.

After deployment and initial configuration of the visual sensor networks, data packets will be transmitted from the source nodes to the sink of the network. A router node will use the SR included in each data packet to forward the packets to the appropriated path. Depending on the number of available transmission paths from a node $h$, different routing policies may be adopted, as expressed in Table II.

TABLE II. SR-BASED ROUTING.

\begin{tabular}{|c|c|}
\hline Path & Group of relevance \\
\hline \multicolumn{2}{|r|}{2 available paths $\left(p_{1}<p_{2}\right)$} \\
\hline$p_{1}$ & Maximum and high relevance packets \\
\hline$p_{2}$ & Medium and low relevance packets \\
\hline \multicolumn{2}{|r|}{3 available paths $\left(p_{1}<p_{2}<p_{3}\right)$} \\
\hline$p_{l}$ & Maximum relevance packets \\
\hline$p_{2}$ & High relevance packets \\
\hline$p_{3}$ & Medium and low relevance packets \\
\hline \multicolumn{2}{|r|}{4 available paths $\left(p_{1}<p_{2}<p_{3}<p_{4}\right)$} \\
\hline$p_{1}$ & Maximum relevance packets \\
\hline$p_{2}$ & High relevance packets \\
\hline$p_{3}$ & Medium relevance packets \\
\hline$p_{4}$ & Low relevance packets \\
\hline \multicolumn{2}{|c|}{5 or more available paths $\left(p_{1}<p_{2}<p_{3}<p_{4}<p_{(4+i)}\right)$} \\
\hline$p_{1}$ & Maximum relevance packets \\
\hline$p_{2}$ & High relevance packets \\
\hline$p_{3}$ & Medium relevance packets \\
\hline$p_{4}$ & Low relevance packets \\
\hline$p_{(4+i)}$ & Backup paths \\
\hline
\end{tabular}

There are four groups of relevance that source nodes may belong to (no packet is transmitted for the irrelevant group). The visual data packets will be routed based on the group of relevance of the transmitting sources (measured through the SR) and the available paths from the router nodes. Each router node will have its own routing policy, according only to its output in terms of transmission paths toward the sink. The backup paths are to be used when the main paths run out of energy, but their usage as redundant paths or for a eventual load balance strategy are out of the scope of this paper.

As heterogeneous sensor networks may be deployed for traditional and visual monitoring, scalar data transmitted from conventional sensor nodes should be routed following the same approach for transmission of low relevance visual data packets.

\section{SIMULATION RESULTS}

We conducted a series of simulations in order to validate the proposed SR-based routing mechanism, estimating for it the average end-to-end delay and the energy consumption in experimental visual sensor networks. The simulations were performed employing the framework Castalia [21], which we adapted to incorporate packet relaying based on the relevance of packets' payloads. Castalia is a C++ discrete event simulator based on the OMNeT++ platform.

The first simulation was concerned with the average endto-end delay of transmission paths, since assumptions (a) and (b) in section III need to be validated. Fig 1 presents the average end-to-end delay according to the number of hops of the path from the source to the sink and the transmission rate, for 1 day of transmissions. For this simulation, we considered that a camera-enabled source node was connected to the sink through a single multihop path, and that the source is transmitting uncompressed $64 \times 64$ 8-bit grayscale still images (4096 bytes plus an image header of 40 bytes). Considering $k=230$ and $\mathrm{z}=23$, each image will be transmitted in 20 data packets. We assume two different transmission frequencies: $f_{s}=2$ and $f_{s}=5$. All nodes are communicating employing a duty-cycle protocol [14].

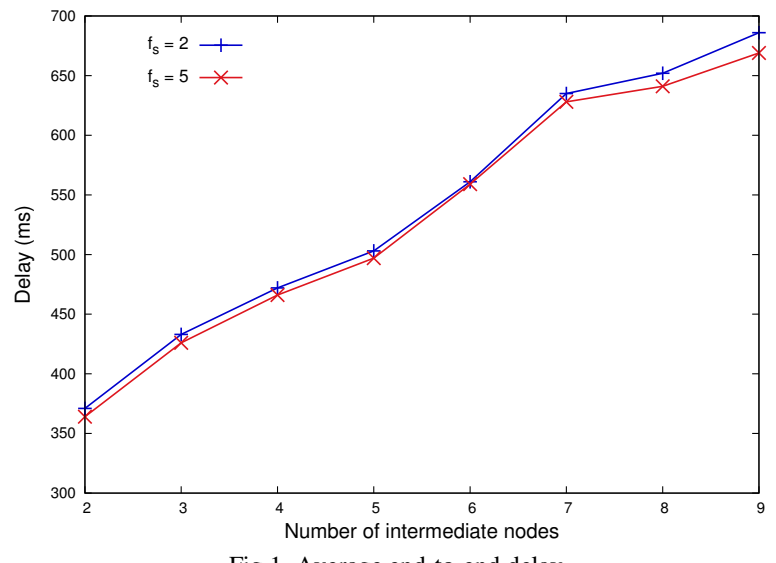

Fig 1. Average end-to-end delay.

It can be seen in Fig 1 that lower delay is achieved for lower transmission rates and for paths with fewer hops, thus supporting our basic assumptions.

Following the proposed SR-based approach, higher relevant packets will be routed through the best transmission paths, following the definitions in Table II. We can then estimate the energy saving when only these packets are routed through the best path, potentially prolonging its transmission lifetime. Fig 2 present a simple network composed of two visual source nodes and two available paths to the sink. We consider that source $\mathrm{s} 1$ is a maximum-relevant source and that $\mathrm{s} 2$ is a lowrelevant source. 


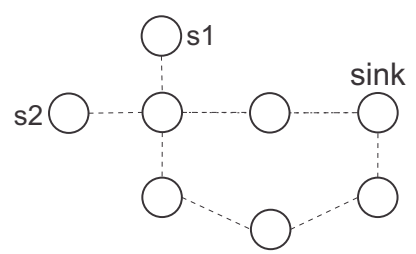

Fig. 2. First communication scenario.

The energy consumption over the best path ( 2 intermediate nodes) for the communication scenario in Fig 2 is presented in Fig 3. The average distance between the nodes is $10 \mathrm{~m}$ and they have a transmission power of $0 \mathrm{dBm}$.

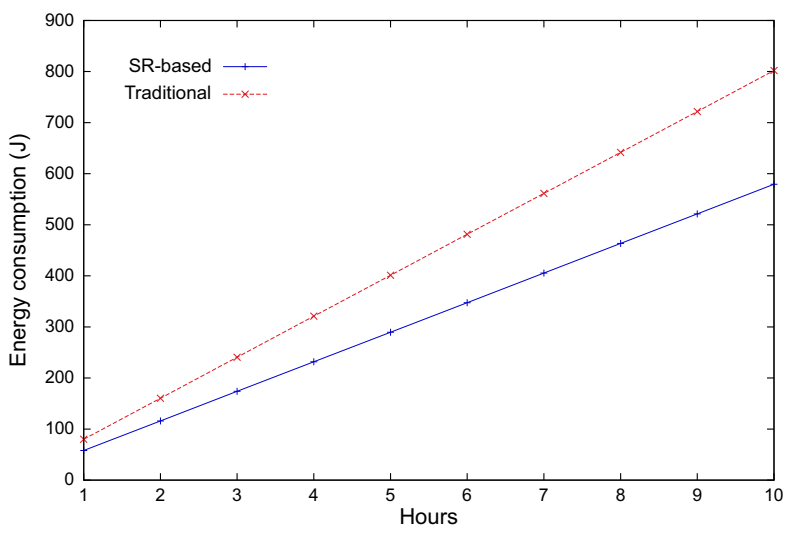

Fig 3. Energy consumption over the best path in Fig 2.

When the SR-based approach is employed, only packets from the highest relevant source will flow through the best path. On the other hand, the traditional transmission mechanism will route all packets through the best path, in order to attend the time-critical requirements of the considered application. In such way, the proposed approach will be more energy-efficient for the best path.

A more complex sensor network is presented in Fig 4. In that communication scenario, there are four source nodes (each one associated to a transmitting group of relevance) connected to the sink through three routing paths.

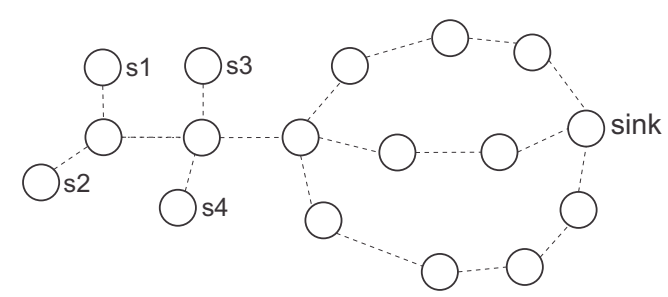

Fig. 4. Second communication scenario.

The energy consumption over the best path (4/5 intermediate nodes) for the communication scenario in Fig 4 is presented in Fig 5. As fewer packets will be routed through the best path, when compared with the traditional transmission approach, less energy is expected to be consumed over it and this claim is also valid for the other paths, following the definitions in Table II.

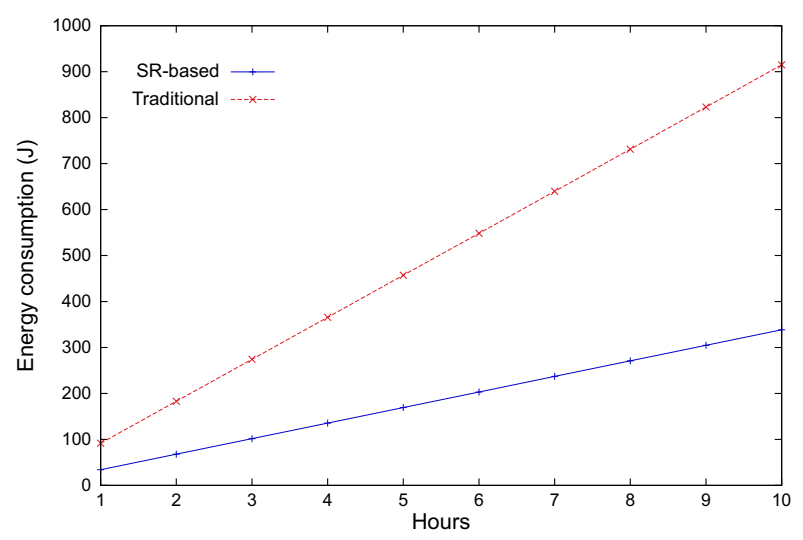

Fig 5. Energy consumption over the best path in Fig 4 .

The final energy saving depends on the network configuration, as the transmission power and the employed communication protocols. Nevertheless, our proposed routing mechanism tends to be the best option when source nodes are differentiated by their sensing relevancies, directing benefiting time-critical applications since the capability of the network to deliver packets in a fast way will be prolonged. Although low-relevant packets will be delayed when routed through slow/large paths, they have lower impact on the monitoring quality. Additionally, timestamp information in transmitted low-relevant data packets may be used to reproduce the received data with temporal correlation with the high-relevant visual information.

Based on the communication scenario in Fig 6, we also assessed the end-to-end delay comparing the SR-based routing approach and a traditional mechanism (all packets are transmitted through the current best path until it is disabled due to energy depletion). We considered a small level of initial energy resources for all nodes in order to outline the path disabling. Note that the network as a whole is consuming almost the same amount of energy whatever is the adopted approach, but for the SR-based mechanism only the highest relevant packets are being routed through the best path, enlarging its lifetime and assuring the lowest average end-toend delay for the data packets that matter most.

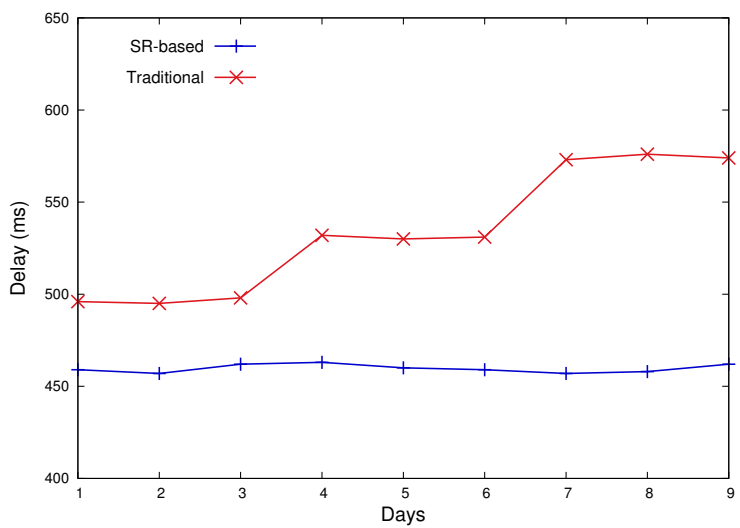

Fig 6. Average end-to-end delay for the scenario in Fig 4. 
In some communication scenarios as the ones presented in Fig 2 and Fig 4, there are nodes that can be a "bottleneck" for the transmission flow from the source nodes to the sink. In fact, the expected lifetime of the network as a whole may vary slightly for both assessed routing approaches. For homogeneous sensor networks where the energy resources of all intermediate nodes are the same, the expected network lifetime may be not significantly influenced by the proposed solution in some cases. However, the results concerning endto-end delay are still significant and valuable for visual sensor networks, once higher traffic incur in higher delay, still enforcing the adoption of the proposed approach.

The success of the proposed approach depends on the network configuration and the sensing relevancies of the source nodes. For example, if most source nodes are established as low-relevant for the application, some paths may be overloaded incurring in congestion and packet loss, while the best paths receive low traffic. In future works, an adaptive mechanism will be designed to balance the traffic considering not only the sensing relevancies of source nodes, but also the maximum throughput of each path.

\section{CONCLUSIONS}

We have proposed a SR-based delay-aware routing mechanism where high relevant packets are transmitted through the shortest paths in order to reduce the end-to-end delay. Doing so, the application monitoring quality is potentially enhanced, directly benefiting time-critical monitoring applications when the best paths are preserved.

This work is not concluded yet and new investigations will be done to further outline the benefits of the proposed routing approach. In fact, there are some relevant issues that need to be properly addressed in future works. As intermediate nodes take their own decision about routing, some looping may be created if the network is not properly configured. More complex sensor network scenarios will be considered when assessing the proposed routing mechanism. Moreover, when better paths relating to end-to-end delay run out of energy, the routing node may change the current routing policy, reconfiguring the way packets are routed over the remaining paths. New simulations will be conducted considering such dynamical behavior of the network. Besides, future works will also consider the assessment of the monitoring quality of the application concerning the monitoring delay.

\section{ACKNOWLEDGMENT}

The authors would like to acknowledge the support of research agencies CAPES (grant no. 9014-11-0) and FCT (project ref. PTDC/EEA-TEL/104185/2008), that partially funded this work.

\section{REFERENCES}

[1] Y. Charfi, B. Canada, N. Wakamiya and M. Murata. "Challenging issues in visual sensor networks". IEEE Wireless Communications, vol. 16, pp. 16, 44-49, 2009.
[2] D. Costa and L. Guedes. "The coverage problem in video-based wireless sensor networks: a survey". Sensors, vol. 10, pp. 8215-8247, 2010 .

[3] I. Almalkawi, M. Zapata, J. Al-Karaki, and J. Morillo-Pozo, "Wireless multimedia sensor networks: current trends and future directions", Sensors, vol. 10, pp. 6662-6717, 2010.

[4] I. Akyildiz, T. Melodia, and K. Chowdhury, "A survey on wireless multimedia sensor networks", Computer Networks, vol. 51, pp. 921960, 2007

[5] S. Qaisar, H. Radha, "Multipath multi-stream distributed reliable video delivery in wireless sensor networks", in Proc. Conference on Information Sciences and Systems, March 2009, pp. 207-212.

[6] V. Lecuire, C. Duran-Faundez and N. Krommenacker, "Energyefficient image transmission in sensor networks", International Journal of Sensor Networks, vol. 4, no. 1-2, pp. 37-47, 2008.

[7] D. Costa and L. Guedes. "Exploiting the sensing relevancies of source nodes for optimizations in visual sensor networks". Multimedia Tools and Applications, in press, 2011.

[8] J. Al-Karaki, A. Kamal. "Routing techniques in wireless sensor networks: a survey", IEEE Wireless Communications, vol. 11, pp. 6-28, 2004.

[9] D. Costa and L. Guedes. "A survey on multimedia-based cross-layer optimization in visual sensor networks". Sensors, vol. 11, pp. 54395468, 2011.

[10] P. Leelapornchai, T. Stockhammer. "Progressive image transmission applying multipath routing in mobile ad hoc networks". in Proc. International Conference on Image Processing, Rochester, MN, USA, September 2002, pp. 553-556.

[11] H. Wang, D. Peng, W. Wang, H. Sharif. "Optimal rate-based image transmissions via multiple paths in wireless sensor network. in Proc. IEEE Multimedia and Expo, Beijing, China, July 2007, pp. 2146-2149.

[12] L. Zhang, M. Hauswirth, L. Shu, Z. Zhou, V. Reynolds, G. Han "Multi-priority multi-path selection for video streaming in wireless multimedia sensor networks". Lecture Notes in Computer Science, vol. 5061, 439-452, 2008

[13] L. Zhou, X. Wang, W. Tu, G. Mutean, and B. Geller, "Distributed scheduling scheme for video streaming over multi-channel multi-radio multi-hop wireless networks," IEEE Journal on Selected Areas in Communications, vol. 28, no. 3, pp. 409-419, 2010.

[14] T. van Dam, K. Langendoe. "An adaptive energy-efficient MAC protocol for wireless sensor networks", In Proc. SenSys, Los Angeles, USA, pp. 1-10, 2003

[15] W. Heinzelman, A. Chandrakasan, H. Balakrishnan, "An applicationspecific protocol architecture for wireless microsensor networks", IEEE Transaction on Wireless Communications, vol 1, no. 4, pp. 660-670, 2002

[16] F. Bajaber, I. Awan, "Adaptive decentralized re-clustering protocol for wireless sensor networks". Journal of Computer and System Sciences, vol 77, pp. 282-292, 2011.

[17] J. Korhonen and Y. Wang. "Effect of packet size on loss rate and delay in wireless links". in Proc. IEEE Wireless Communications and Networking Conference, New Orleans, USA, pp. 1608-1613, 2005.

[18] D. Costa, L. Guedes, A discrete wavelet transform (DWT)-based energy-efficient selective retransmission mechanism for wireless image sensor networks, Journal of Sensor and Actuator Networks, vol. 1, no. 1, pp. 3-35, 2012.

[19] D. Costa, L. Guedes, F. Vasques, P. Portugal, "Effect of frame size on energy consumption in wireless image sensor networks", in Proc. IEEE Imaging Systems and Techniques, Manchester, United Kingdom, 2012.

[20] L. Shu, Z.B. Zhou, M. Hauswirth, D.L. Phuoc, P. Yu, L. Zhang, "Transmitting streaming data in wireless multimedia sensor networks with holes". in Proc. International Conference on Mobile and Ubiquitous Multimedia, Oulu, Finland, 2007.

[21] Y. Tselishchev, A. Boulis and L. Libman, "Experiences and lessons from implementing a wireless sensor network MAC protocol in the Castalia simulator". In Proc. IEEE Wireless Communications \& Networking Conference, Sydney, Australia, pp. 1-6, 2010. 